\title{
Complete mediastinal lymph node dissection versus systematic lymph node sampling in surgical treatment of non-small cell lung cancer: do we have the answer?
}

\author{
Yuzhao Wang, Gail E. Darling \\ Division of Thoracic Surgery, Department of Surgery, Toronto General Hospital, University Health Network, University of Toronto, Toronto, Canada \\ Correspondence to: Gail E. Darling, MD, FRCSC. Division of Thoracic Surgery, Department of Surgery, Toronto General Hospital, University Health \\ Network, University of Toronto, 200 Elizabeth St, 9N-955, Toronto, Ontario M5G 2C4, Canada. Email: gail.darling@uhn.ca. \\ Provenance: This is an invited Editorial commissioned by Section Editor Dr. Min Zhang (Department of Thoracic Oncology, The First Affiliated \\ Hospital of Chongqing Medical University, Chongqing, China). \\ Comment on: Mokhles S, Macbeth F, Treasure T, et al. Systematic lymphadenectomy versus sampling of ipsilateral mediastinal lymph-nodes during \\ lobectomy for non-small-cell lung cancer: a systematic review of randomized trials and a meta-analysis. Eur J Cardiothorac Surg 2017;51:1149-56.
}

Submitted Sep 14, 2017. Accepted for publication Oct 05, 2017.

doi: $10.21037 /$ jtd.2017.10.39

View this article at: http://dx.doi.org/10.21037/jtd.2017.10.39

The question regarding the standard approach to mediastinal lymph nodes at time of pulmonary resection for non-small cell lung cancer has been discussed for several decades. Some argue that complete mediastinal lymph node dissection contributes to more accurate lymph node staging and survival benefit, whereas others believe that systematic mediastinal lymph node sampling is adequate for accurate staging and that formal dissection of the mediastinal nodes does not provide any survival advantage as patients with positive $\mathrm{N} 2$ nodes die from systemic disease.

In the recent review written by Mokhles and colleagues (1), five randomized controlled trials addressing this controversy during the past 20 years were evaluated. Although the authors report improved long term survival with mediastinal lymph node dissection compared with sampling based on their meta-analysis, they comment that this result is not conclusive because of bias in these trials. Nevertheless, there are aspects of these trials which warrant discussion.

It is acknowledged that accuracy of lymph node staging is important to guide treatment and predict prognosis. The first question is whether mediastinal lymph node dissection detects more pathological N2 disease than systematic lymph node sampling in patients with clinically negative nodes prior to resection? In the American College of Surgeons Oncology Group (ACOSOG) Z0030 trial (2), occult N2 disease was detected by dissection in only $4 \%(21 / 525)$ of the cases that had been proven to be N0 disease by systematic sampling. However, two points should be emphasized. First, the enrolled cases of trial Z0030 included clinical T1 or T2 tumor only, therefore it is unknown whether this result would apply to patients with higher T stage. In theory, N2 disease should be more prevalent in higher $\mathrm{T}$ stage disease and therefore should be more easily detected by sampling, but though there is insufficient evidence to prove this. Secondly, in these studies, PET-CT was not used except in ACOSOG Z0030 where it was used only in the latter part of the trial. Also except for ACOSOG Z0030, there was low utilization of mediastinoscopy and no EBUS or EUS or VATS staging. These factors undoubtedly reduced the accuracy of preresection staging. With less accurate pre-resection staging, removal of mediastinal nodes at the time of resection becomes more important for accurate staging.

In ACOSOG Z0030, a rigorous protocol of systematic sampling was followed: for tumors in the right lung, stations $2 \mathrm{R}, 4 \mathrm{R}, 7$, and $10 \mathrm{R}$ was sampled; for tumors in the left lung, stations 5, 6, 7, and 10L should be sampled. Based on this trial, it is can be concluded that either systematic sampling or mediastinal lymph node dissection is adequate for lymph node staging for $\mathrm{T} 1$ or $\mathrm{T} 2$ tumors.

More controversial is whether mediastinal lymph node dissection improves survival. Among the five trials, two found better survival in patients receiving mediastinal lymph node dissection than those receiving sampling, whereas the other three did not. Why the discrepancy? First, there is 
significant heterogeneity in the stage of disease amongst these trials. These papers suggest that there may be a survival benefit of mediastinal lymph node dissection for patients with more advanced disease but it is not known whether this is due to the removal of metastatic lymph nodes or due to subsequent treatment given because of positive mediastinal nodes identified. Second, it should be noted that the trials reporting a survival benefit included patients with higher stage disease and in whom pre-resection staging was not performed. In ACOSOG Z0030, even though mediastinal dissection identified $4 \%$ of patients had N2 disease that was not identified by systematic sampling, there was no survival difference between the two groups.

ACOSOG Z0030 trial is the largest, as well as the only multicenter one in the five trials, in which all patients were clinical $\mathrm{T} 1$ or $\mathrm{T} 2$ tumors and had been confirmed as N0 disease by rigorous systematic sampling before randomization (2). In this trial, the authors found that mediastinal lymph node dissection did not improve either overall or disease-free survival compared with sampling. However, this result is easily misinterpreted as a suggestion that mediastinal lymph node dissection is unnecessary for T1 and T2 disease. It is should be emphasized that lymph node dissection during resection may be omitted only if rigorous systematic sampling by mediastinoscopy, EBUS and/or EUS is performed prior to resection to confirm lymph nodes to be pathologically negative. The authors of Z0030 trial indicated that if invasive mediastinal staging (IMS) is not performed prior to surgery or the criterion of systematic sampling is not complied with, mediastinal lymph node dissection is still be recommended.

Although the authors have completed an excellent review, we are still left without a conclusive answer. A randomized clinical trial using modern staging with PET-CT prior to invasive staging with adequate power would be more helpful for guiding treatment for each specific patient we meet in the future. The ongoing Japanese Clinical Oncology Group trial (JCOG 1413) which randomizes patients with clinical stage I and II based on PET-CT to either mediastinal lymph node dissection or lobe specific lymph node sampling should provide evidence that will help to answer this question (3). Until we have the results of that trial, our guidance should be to do a mediastinal lymphadenectomy to optimize staging and local control and to guide adjuvant therapy except in the case of an early lung cancer ( $\mathrm{T} 1$ or $\mathrm{T} 2$ ), when a systematic lymph node sampling is adequate based on the ACOSOG Z0030 trial.

Clinical practice guidelines recommend IMS for nonsmall cell lung cancer except for small (T1) peripheral tumors (4). IMS includes mediastinoscopy, EBUS-TBNA and EUS. Despite these recommendations, the utilization of IMS remains low (5). Given these practice patterns, complete mediastinal lymph node dissection should be performed.

\section{Acknowledgements}

None.

\section{Footnote}

Conflicts of Interest: The authors have no conflicts of interest to declare.

\section{References}

1. Mokhles S, Macbeth F, Treasure T, et al. Systematic lymphadenectomy versus sampling of ipsilateral mediastinal lymph-nodes during lobectomy for nonsmall-cell lung cancer: a systematic review of randomized trials and a meta-analysis. Eur J Cardiothorac Surg 2017;51:1149-56.

2. Darling GE, Allen MS, Decker PA, et al. Randomized trial of mediastinal lymph node sampling versus complete lymphadenectomy during pulmonary resection in the patient with N0 or N1 (less than hilar) non-small cell carcinoma: results of the American College of Surgery Oncology Group Z0030 Trial. J Thorac Cardiovasc Surg 2011;141:662-70.

3. A randomized phase III trial of lobe-specific vs. systematic nodal dissection for clinical stage I-II non-small cell lung cancer (Japan Clinical Oncology Group 1413). Available online: https://upload.umin.ac.jp/cgi-open-bin/ctr/ctr_ view.cgi? recptno=R000028940

4. Darling G, Dickie J, Malthaner R, et al. Invasive Mediastinal Staging of Non-Small Cell Lung Cancer. Cancer Care Ontario Program in Evidence Based Care \#17-6. 2010. Available online: http://www.cancercare.on.ca/toolbox/ qualityguidelines/clin-program/surgery-ebs/

5. Bendzsak A, Waddell TK, Yasufuku K, et al. Invasive mediastinal staging guideline concordance. Ann Thorac Surg 2017;103:1736-41.

Cite this article as: Wang Y, Darling GE. Complete mediastinal lymph node dissection versus systematic lymph node sampling in surgical treatment of non-small cell lung cancer: do we have the answer? J Thorac Dis 2017;9(11):41694170. doi: $10.21037 /$ jtd.2017.10.39 